\title{
Predictive value of lymphocyte-to-monocyte ratio (LMR) and neutrophil-to-lymphocyte ratio (NLR) in patients with oesophageal cancer undergoing concurrent chemoradiotherapy
}

\author{
Ke-Jie Li ${ }^{1}$, Xiao-Fang Xia', Meng Su${ }^{2}$, Hui Zhang ${ }^{1}$, Wen-Hao Chen ${ }^{1}$ and Chang-Lin Zou²*
}

\begin{abstract}
Background and objectives: The survival rate of patients with advanced oesophageal cancer is very low and can vary significantly, even among patients with the same TNM stage. It is important to look for indicators that are economical and readily available to predict overall survival. The aim of this study was to determine whether lymphocyte-to-monocyte ratio (LMR) and neutrophil-to-lymphocyte ratio (NLR) could be potential predictors of survival in patients with advanced oesophageal squamous cell carcinoma (ESCC) undergoing concurrent chemoradiotherapy.
\end{abstract}

Methods: Differences in survival among 204 patients with advanced oesophageal cancer who underwent concurrent chemoradiotherapy were collected and analysed. Univariate and multivariate COX regression analyses were used to investigate the association between blood inflammatory markers and patient survival before treatment.

Results: Univariate COX regression analyses showed that a history of alcohol use, neutrophil count, LMR, NLR, tumour length, and $\mathrm{N}$ stage were significantly associated with the survival of tumour patients receiving concurrent chemoradiotherapy. Multivariate COX regression analysis showed that NLR and LMR were predictors of outcome in tumour patients receiving chemoradiotherapy. According to receiver operating characteristic (ROC) curve analysis, the AUC of LMR and NLR was 0.734 and 0.749, and the best cutoff point for LMR and NLR was 3.03 and 2.64, respectively.

Conclusions: LMR and NLR can be used to predict the survival of patients with advanced oesophageal cancer receiving concurrent chemoradiotherapy, thereby providing clinicians with suggestions for further treatment options.

Keywords: Oesophageal cancer, Blood inflammatory markers, Concurrent chemoradiotherapy, Overall survival

\footnotetext{
* Correspondence: zcl19670115@163.com

${ }^{2}$ Department of Radiotherapy and Chemotherapy, The First Affiliated Hospital

of Wenzhou Medical University, WenZhou, China

Full list of author information is available at the end of the article
}

(c) The Author(s). 2019 Open Access This article is distributed under the terms of the Creative Commons Attribution 4.0 International License (http://creativecommons.org/licenses/by/4.0/), which permits unrestricted use, distribution, and reproduction in any medium, provided you give appropriate credit to the original author(s) and the source, provide a link to the Creative Commons license, and indicate if changes were made. The Creative Commons Public Domain Dedication waiver (http://creativecommons.org/publicdomain/zero/1.0/) applies to the data made available in this article, unless otherwise stated. 


\section{Background}

Oesophageal cancer is one of the most common tumours in the world. According to the latest statistics, the incidence of oesophageal cancer ranks fourth among all tumours [1]. There are two main pathological types of oesophageal cancer: oesophageal squamous cell carcinoma (ESCC) and oesophageal adenocarcinoma (EAC), and ESCC is the more common type, especially in Asia and Africa [2]. Due to the lack of specific symptoms in the early stage of oesophageal squamous cell carcinoma, most patients are in the advanced stage at the time of diagnosis and lose the opportunity for surgery. In China, approximately 38,000 people die of oesophageal cancer each year [3].

For patients with inoperable oesophageal cancer, there are a variety of treatments, including radiotherapy, chemotherapy, concurrent chemoradiotherapy, targeted therapy and immunotherapy [4]. In clinical work, concurrent chemoradiotherapy is the main means we use to improve the survival of patients with advanced oesophageal cancer. Radiation therapy has played an increasingly important role in the treatment of oesophageal cancer [5]. With the continuous updating and development of technology, techniques such as intensity-modulated radiation therapy (IMRT), volumetric arc therapy (VMAT) and proton therapy have been used to treat patients with advanced oesophageal cancer. Studies by Stefania Martini and Francesca Arcadipane et al. have demonstrated that volumetric modulated arc therapy (VMAT) is an effective and safe strategy for treatment of patients with advanced oesophageal cancer [6-8]. According to a study by Samantha Warren et al., proton therapy for patients with advanced oesophageal cancer may have lower blood toxicity [9]. Similarly, a retrospective analysis by Mian $\mathrm{Xi}$ and $\mathrm{Cai} \mathrm{Xu}$ et al. showed that the metrological advantages of proton therapy play an important role in improving the overall survival of patients with advanced oesophageal cancer [10]. These methods can not only improve the therapeutic effect on the tumour area but also better protect normal tissues, such as the heart and lungs. However, the therapeutic effects of these treatments in individuals with oesophageal cancer remain uncertain. Because each individual patient is different, even if their clinical TNM staging is the same, their survival can vary greatly after receiving similar treatment. We have observed that some stage IV patients can continue to survive for several years, and some patients die within a few months after diagnosis. We expect a positive treatment response for patients who expect to live longer and seek prolong their survival as much as possible. For patients who are expected to have a shorter survival time, some palliative treatments can be used to alleviate suffering and avoid the side effects and financial burden caused by overly aggressive treatment. Recently, some studies have shown that certain protein markers or genes can predict the survival of patients with oesophageal cancer [11, 12], but the acquisition of these indicators is very cumbersome, imposes a large economic burden and requires an extended wait time for patients. Therefore, it is especially important to identify blood inflammatory markers that are easy to obtain and can predict patient survival.

Currently, inflammatory markers such as LMR and NLR are widely studied in predicting the prognosis of oesophageal cancer. Based on their research, Dawei Yuan and colleagues believe that NLR has great value in predicting the disease-free survival and overall survival of patients with oesophageal cancer after surgery [13]. Noriyuki Hirahara and his team used a scoring system based on LMR, NLR and PLR and reported that LMR and NLR are effective predictors of overall survival in patients with oesophageal cancer [14]. Conway AM and Salih Z et al. concluded that NLR is an independent prognostic factor for oesophageal cancer and can be used in conjunction with AJCC8 clinical staging to predict baseline prognosis stratification in patients newly diagnosed with resectable, oesophageal adenocarcinoma [15]. The above studies suggest that LMR and NLR are potential clinical biomarkers that are easy to calculate, can be repeatably obtained and have a low cost. However, there have been no reports on the correlation between inflammation-related indicators and the overall survival of oesophageal cancer patients after they receive radiotherapy and chemotherapy. The aim of our study was to determine whether LMR and NLR could be predictors of survival in patients with advanced oesophageal cancer that receive concurrent chemoradiotherapy.

\section{Materials and methods \\ Patient selection}

The study included 204 patients with advanced oesophageal cancer treated at the First Affiliated Hospital of Wenzhou Medical University between 2010 to 2014. The patients were diagnosed with advanced oesophageal cancer at the time of diagnosis and thus had lost the best opportunity for surgery. The inclusion conditions were as follows: (1) patients between 18 and 85 years of age; (2) pathologically confirmed oesophageal squamous cell carcinoma; (3) only received concurrent chemoradiotherapy after diagnosis; (4) exhibited no significant adverse effect on blood inflammation diseases, such as vasculitis and systemic lupus erythaematosus. We collected blood inflammatory markers from these patients prior to treatment, including white blood cells, neutrophils, monocytes, lymphocytes, LMR, NLR, NMR, and CEA. In addition, some basic characteristics of the cancer patients, such as age, gender, smoking history, drinking history, and ECOG score, were recorded, as well as tumour features, including pathological type, degree of 
differentiation, lymph node metastasis and tumour location, length, and width, which were obtained from CT, endoscopic ultrasound, and pathological diagnosis.

\section{Treatment protocol}

All patients enrolled received concurrent chemoradiotherapy. A total dose of up to $54 \mathrm{~Gy}$ was delivered via standard fractionated radiotherapy in 30 fractions (on work days; 1.8 Gy per fraction; over a 6 -week cycle). The principle of target mapping is as follows: GTV represents the primary oesophageal lesion and shows the length of the tumour as indicated by oesophageal angiography and/or oesophagoscopy and/or intraluminal ultrasound. The length of the primary tumour is combined with $\mathrm{CT}$ and PET/CT imaging results and the scope of invasion. An enlarged metastatic lymph node is represented by GTVnd: a positive lymph node is defined as a lymph node with the largest short diameter $\geq 1 \mathrm{~cm}$ detected by CT/MRI or a node with a size not more than $1 \mathrm{~cm}$ but showing obvious necrosis and ring enhancement. CTV includes GTV and the GTVnd+ lymph node drainage area and an additional $0.8 \mathrm{~cm}$ outside GTV and GTVnd (plane), $3-5 \mathrm{~cm}$ in GTV and GTVnd (up and down), or 1.5 on the CT level with lymph node metastasis $-2.0 \mathrm{~cm}$. The PTV is $0.5 \mathrm{~cm}$ on the basis of CTV. The concurrent chemotherapy regimen was paclitaxel $135 \mathrm{mg} / \mathrm{m} 2$ combined with cisplatin $25 \mathrm{mg} / \mathrm{m} 2$ once every 3 weeks per cycle. The study was approved by the Ethics Committee of the First Hospital affiliated with Wenzhou Medical University. Because all the patients in this retrospective study had died, informed consent was obtained from their family members or their pretreatment authorized recipients.

\section{Evaluation strategy}

The primary end point of assessment was patient overall survival (OS), which was defined as the time from randomization to the time of death from any cause. For subjects who had missed their follow-up visits prior to death, their last follow-up was counted as the time of death. Secondary assessment endpoints were progression-free survival (PFS) and objective response rate (ORR). PFS was defined as the time between the onset of randomization and the progression (or any aspect) of tumourigenesis or death (for any reason). The ORR was defined as the proportion of patients whose tumour volume was reduced to a predetermined value and was maintained for the minimum time limit, which was the sum of complete and partial relief. In other words, ORR $=\mathrm{CR}+\mathrm{PR}$. CR indicates that the tumour completely disappeared for more than 1 month; PR indicates that the sum of the largest diameters of the tumour was reduced by at least $30 \%$ and was maintained for at least 4 weeks.

\section{Data statistics}

All statistical analyses were performed using a social science statistical software package, version 22.0 (SPSS Inc., Chicago, IL, USA). A receiver operating characteristic curve was used to select the best cutoff value for blood inflammatory indicators and to stratify the indicators. The Kaplan-Meier method was used to plot survival curves. A chi-square test was used to analyse correlations between predictors and tumour parameters. The routine parameters included haematology markers (white blood cells, neutrophils, monocytes, lymphocytes, LMR, NLR, NMR, PLR, and CEA) and clinical pathology characteristics (sex, age, drinking history, tumour site, tumour stage, and ECOG score). Univariate analysis was performed to determine which variables were associated with the survival of the tumour patient. Multivariate COX regression analysis was used to identify predictors of advanced oesophageal cancer. $p<0.05$ was considered statistically significant.

\section{Result}

A total of 204 patients with oesophageal cancer were included in this study. The age distribution of the patients was between 38 and 85 years, with a median age of 65.8 years; other specific clinical and pathological features of the patients are shown in Table 1.

The median follow-up time was 11.5 months (range: 2.1 to 77.4 months). According to univariate COX regression (Table 2), drinking history, tumour length, neutrophils, NLR, and LMR were associated with survival in patients undergoing concurrent chemoradiotherapy for oesophageal cancer.

Multivariate COX regression (Table 3) analysis was performed with the statistically significant indicators in the univariate COX regression analysis, and the results suggested that NLR (OR 2.233 (95\% CI 1.67-2.96), $p<$ 0.005), LMR (OR 0.278 (95\% CI 0.205-0.376), $p<0.05$ ), and $\mathrm{T}$ stage are predictors of survival in patients with oesophageal cancer. Patients with high LMR values showed longer survival than patients with low LMR values, whereas patients with a lower NLR had a longer survival period than patients with high NLR values.

A receiver operating characteristic (ROC) curve (Fig. 1) was plotted to assess the value of statistically significant variables in the COX regression model for NLR and LMR. The area under the curve of LMR and NLR was 0.734 and 0.749 , and the optimal cutoff value of LMR and NLR was 3.03 and 2.64, respectively. The ROC curve is shown in Fig. 1.

Kaplan-Meier analysis was applied to construct a survival curve. In patients with an NLR (Fig. 2) less than 2.64, the mean survival was 19.8 months, and the median survival was 15 months. In patients with an NLR greater than or equal to 2.64 , the mean survival was 10.3 
Table 1 Basic physiological and physiological characteristics of 204 patients

\begin{tabular}{|c|c|}
\hline Characteristic & No of people(\%) \\
\hline No of people(\%) & 204 \\
\hline \multicolumn{2}{|l|}{ Sex } \\
\hline Female & $33(16.2 \%)$ \\
\hline Male & $171(83.8 \%)$ \\
\hline \multicolumn{2}{|l|}{ Age } \\
\hline Median & 65.8 years \\
\hline Range & $38-85$ \\
\hline 65 years old or older & $102(50 \%)$ \\
\hline Under 65 years old & $102(50 \%)$ \\
\hline \multicolumn{2}{|l|}{ History of smoking } \\
\hline Yes & $104(50.9 \%)$ \\
\hline No & $100(49.1 \%)$ \\
\hline \multicolumn{2}{|l|}{ Drinking history } \\
\hline Yes & $100(49.1 \%)$ \\
\hline No & $104(50.9 \%)$ \\
\hline \multicolumn{2}{|l|}{ Differentiation } \\
\hline Highly differentiation & $51(25 \%)$ \\
\hline Medium differentiation & $96(47.1 \%)$ \\
\hline Low differentiation & $57(27.9 \%)$ \\
\hline \multicolumn{2}{|l|}{ Tumor site } \\
\hline Upper thoracic portion & $102(50 \%)$ \\
\hline Middle thoracic portion & $78(38.2 \%)$ \\
\hline Low thoracic portion & $24(11.8 \%)$ \\
\hline \multicolumn{2}{|l|}{ Tumor length (cm) } \\
\hline Median & $4.8 \mathrm{~cm}$ \\
\hline Range & $0.9-11.3 \mathrm{~cm}$ \\
\hline More than $4 \mathrm{~cm}$ & $104(50.9 \%)$ \\
\hline Less than $4 \mathrm{~cm}$ & $100(49.1 \%)$ \\
\hline \multicolumn{2}{|l|}{ T-staging } \\
\hline $\mathrm{T} 1+\mathrm{T} 2$ & $71(34.8 \%)$ \\
\hline $\mathrm{T} 3+\mathrm{T} 4$ & $133(65.2 \%)$ \\
\hline \multicolumn{2}{|l|}{ N-staging } \\
\hline NO & 75 (36.8\%) \\
\hline $\mathrm{N} 1+\mathrm{N} 2$ & $129(63.2 \%)$ \\
\hline \multicolumn{2}{|l|}{ ECOG score } \\
\hline 0 point & $174(85.3 \%)$ \\
\hline 1 point & $21(10.3 \%)$ \\
\hline 2 point & $9(4.4 \%)$ \\
\hline
\end{tabular}

months, and the median survival was 8 months. In patients with an LMR (Fig. 3) less than 3.03, the mean survival was 8.3 months, and the median survival was 7 months. In patients with an LMR greater than or equal
Table 2 Univariate COX regression analysis of the relationship between pathophysiological parameters and survival time of patients

\begin{tabular}{llll}
\hline Parameter & OR & $95 \% \mathrm{Cl}$ & $P$ \\
\hline Sex & 1.121 & $0.770-1.631$ & 0.551 \\
Age & 1.119 & $0.848-1.476$ & 0.427 \\
Smoking history & 1.141 & $0.864-1.506$ & 0.352 \\
Drinking history & 1.333 & $1.005-1.769$ & 0.046 \\
Differentiation & 0.972 & $0.715-1.321$ & 0.856 \\
Tumor site & 0.858 & $0.647-1.137$ & 0.286 \\
Tumor length & 1.365 & $1.033-1.802$ & 0.028 \\
Tumor width & 1.154 & $0.876-1.520$ & 0.309 \\
T-staging & 1.468 & $1.095-1.969$ & $<0.05$ \\
N-staging & 1.707 & $1.271-2.294$ & $<0.05$ \\
ECOG score & 1.173 & $0.974-1.731$ & 0.423 \\
Lymphocytes & 0.896 & $0.677-1.186$ & 0.443 \\
Neutrophils & 1.545 & $1.162-2.053$ & 0.003 \\
Monocytes & 1.176 & $0.891-1.574$ & 0.252 \\
LMR & 0.278 & $0.205-0.376$ & $<0.05$ \\
NLR & 2.223 & $1.670-2.960$ & $<0.05$ \\
CEA & 0.916 & $0.666-1.260$ & 0.590 \\
NMR & 0.900 & $0.591-1.370$ & 0.622 \\
\hline
\end{tabular}

to 3.03 , the mean survival was 20.2 months, and the median survival was 16 months. Both $p$ values were less than 0.05 , indicating statistical significance.

Chi-square tests were used to analyse the relationship between LMR and NLR and conventional tumour parameters. Analyses showed that NLR (Table 4) was associated with $\mathrm{N}$ stage $(p<0.05)$, tumour location $(p=0.017)$, tumour stage $(\mathrm{p}<0.05)$, and treatment efficacy ( $\mathrm{p}<0.05)$, while LMR (Table 5) was associated with efficacy of treatment $(p<0.05)$. At the same time, multivariate logistic regression showed that NLR (OR 1.918 (95\% CI 1.406-2.617) $p<0.05)$, and LMR (OR 0.337 (95\% CI 0.245-0.463) $p<0.05$ ) were significantly associated with PFS.

Table 3 Multivariate COX regression analysis of the relationship between clinical variables and patient survival

\begin{tabular}{llll}
\hline Parameter & $\mathrm{OR}$ & $95 \% \mathrm{Cl}$ & $P$ \\
\hline Drinking history & 1.103 & $0.813-1.497$ & 0.528 \\
Tumor length & 0.880 & $0.611-1.268$ & 0.492 \\
T-staging & 1.598 & $1.037-2.462$ & 0.034 \\
N-staging & 0.957 & $0.687-1.333$ & 0.957 \\
Neutrophils & 1.197 & $0.880-1.628$ & 0.253 \\
LMR & 0.331 & $0.238-0.459$ & $<0.05$ \\
NLR & 1.597 & $1.151-2.215$ & $<0.05$ \\
\hline
\end{tabular}




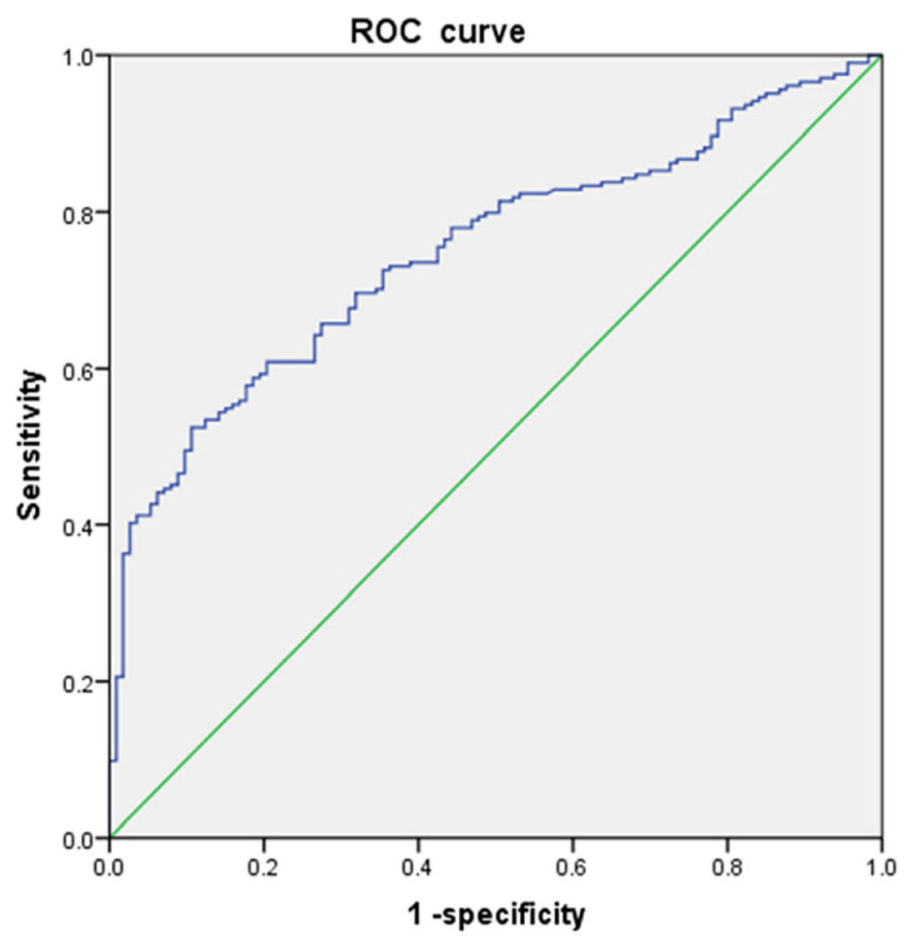

The diagonal segment generated by the ties.

(a)

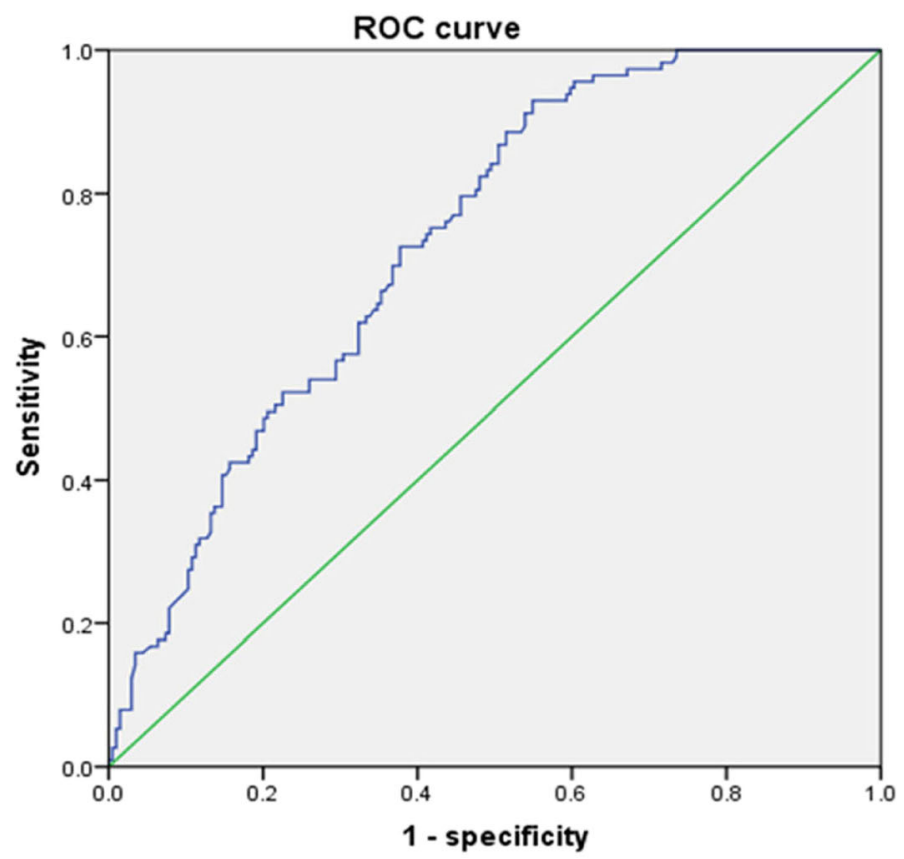

The diagonal segment generated by the ties.

(b)

Fig. 1 Receiver operating characteristic (ROC) curve plotted to determine the value of a statistically significant variable in the COX regression model for NLR (a) and LMR (b). According to ROC analysis, the area under the curve of NLR and LMR was 0.749 and 0.734 , respectively, and the optimal cutoff point was 2.64 and 3.03 , respectively 


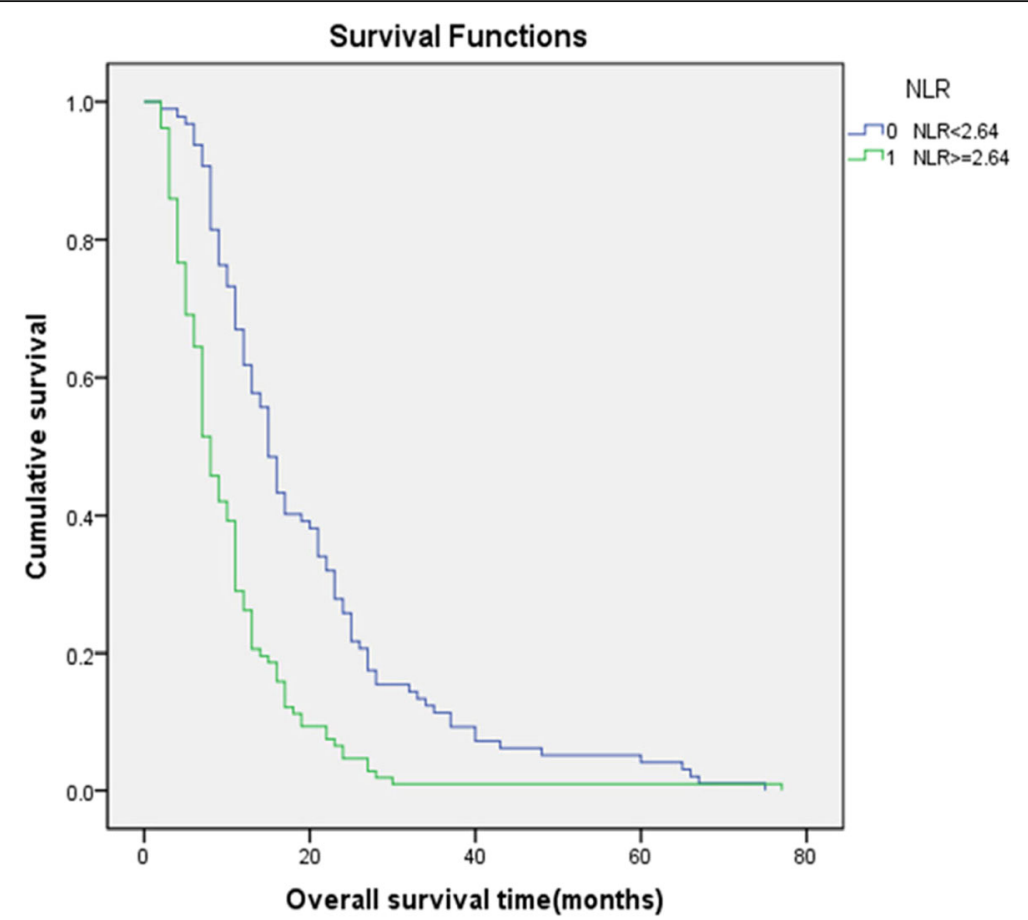

Fig. 2 Kaplan-Meier survival curves for patients with advanced oesophageal cancer in different NLR groups. The blue curve represents the overall survival of patients with an NLR less than 2.64, while the green curve represents the overall survival of patients with an NLR greater than or equal to 2.64. The mean survival time of patients in the low NLR group was 19.8 months, and the mean survival time of patients in the high NLR group was 10.3 months, with a $p<0.05$, indicating a significant difference between the two groups

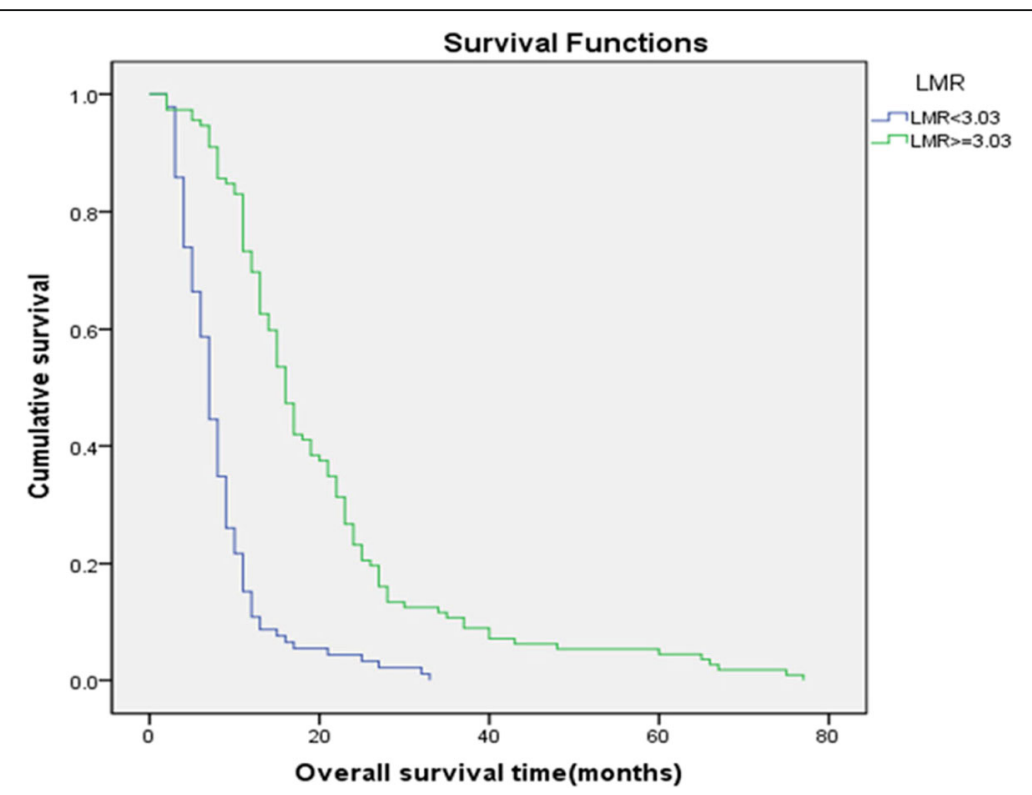

Fig. 3 Kaplan-Meier survival curves for patients with advanced oesophageal cancer in different LMR groups. The blue curve represents the overall survival of patients with an LMR less than 3.03, while the green curve represents the overall survival of patients with an LMR greater than or equal to 3.03. The mean survival time of patients in the group with an LMR less than 3.03 was 8.3 months, while that of patients in the group with an LMR greater than or equal to 3.03 was 20.2 months, with a $p$ value less than 0.05 , indicating a significant difference between the two groups 
Table 4 Association of pathological features and NLR in patients

\begin{tabular}{|c|c|c|c|}
\hline Characteristic, $n=204$ & $\mathrm{NLR}<2.64$ & NLR $>=2.64$ & $P$ \\
\hline \multicolumn{4}{|l|}{$\mathrm{N}$-staging } \\
\hline NO & 26 & 49 & \\
\hline $\mathrm{Nl}+\mathrm{N} 2$ & 71 & 58 & $<0.05$ \\
\hline \multicolumn{4}{|l|}{ Treatment efficacy } \\
\hline$C R+P R$ & 63 & 34 & \\
\hline$S D+P D$ & 34 & 73 & $<0.05$ \\
\hline \multicolumn{4}{|l|}{ T-staging } \\
\hline $\mathrm{T} 1+\mathrm{T} 2$ & 38 & 33 & \\
\hline $\mathrm{T} 3+\mathrm{T} 4$ & 59 & 74 & 0.212 \\
\hline \multicolumn{4}{|l|}{ Tumor location } \\
\hline Upper thoracic & 40 & 62 & \\
\hline Lower thoracic & 57 & 45 & 0.017 \\
\hline \multicolumn{4}{|l|}{ Age } \\
\hline$<65$ years old & 48 & 54 & \\
\hline$>=65$ years old & 49 & 53 & 0.889 \\
\hline \multicolumn{4}{|l|}{ Sex } \\
\hline Male & 79 & 92 & \\
\hline Female & 18 & 15 & 0.066 \\
\hline \multicolumn{4}{|l|}{ Drinking history } \\
\hline Yes & 41 & 59 & \\
\hline No & 56 & 48 & 0.379 \\
\hline \multicolumn{4}{|l|}{ Differentiation } \\
\hline Medium-high differentiation & 71 & 76 & \\
\hline Low differentiation & 26 & 31 & 0.73 \\
\hline
\end{tabular}

\section{Discussion}

Oesophageal cancer is a malignant tumour with high incidence. Its occurrence and development are related to age, gender, occupation, race, region, living environment, eating habits, and genetic susceptibility, among other factors. Long-term drinking of strong alcohol, habitual smoking, eating food that is too hard, overheating, eating too fast can cause irritation, and chronic inflammation can lead to oesophageal cancer. Despite the continuous innovation in surgical methods, improvement in chemotherapy regimens, adjustment of radiotherapy plans, and continuous development and marketing of targeted therapeutics and immunotherapeutics, the survival rate of patients with advanced oesophageal cancer is still very low. Moreover, patients with oesophageal cancer with the same TMN stage have widely varying survival outcomes after receiving similar treatment, and some patients even rapidly deteriorate after treatment with standardized chemotherapy. Therefore, predicting a patient's likely survival before treatment can help the clinician determine prognosis and provide individualized treatment. Currently, clinical TNM staging is considered the gold standard for predicting
Table 5 Association of pathological features and LMR in patients

\begin{tabular}{|c|c|c|c|}
\hline Characteristic, $n=204$ & LMR $<3.03$ & LMR $>=3.03$ & $P$ \\
\hline \multicolumn{4}{|l|}{ Sex } \\
\hline Male & 77 & 94 & \\
\hline Female & 15 & 18 & 0.964 \\
\hline \multicolumn{4}{|l|}{ Age } \\
\hline$<65$ years old & 49 & 53 & \\
\hline$>=65$ years old & 43 & 59 & 0.399 \\
\hline \multicolumn{4}{|l|}{$\mathrm{N}$-staging } \\
\hline No & 39 & 36 & \\
\hline $\mathrm{NI}+\mathrm{N} 2$ & 53 & 76 & 0.131 \\
\hline \multicolumn{4}{|l|}{ Treatment efficacy } \\
\hline$C R+P R$ & 26 & 71 & \\
\hline$S D+P D$ & 66 & 41 & $<0.05$ \\
\hline \multicolumn{4}{|l|}{ Drinking history } \\
\hline Yes & 42 & 58 & \\
\hline NO & 50 & 54 & 0.402 \\
\hline \multicolumn{4}{|l|}{ Differentiation } \\
\hline Medium-high differentiation & 65 & 82 & \\
\hline Low differentiation & 27 & 30 & 0.685 \\
\hline
\end{tabular}

outcomes and determining treatment options. However, because accurate TNM staging requires post-operative pathology, it is difficult for TNM staging to predict survival and determine further treatment strategies for patients with advanced oesophageal cancer who are inoperable. In view of the above facts, there is an urgent need to explore prognostic biomarkers that are easily evaluated and reproducible for patients with advanced inoperable oesophageal cancer.

Among the many potential biomarkers, such as genetic, immunological, and haematological markers, systemic inflammatory markers in peripheral blood are receiving increasing attention for prediction of tumour recurrence, metastasis, and prognosis [16-18]. Recent studies have shown that absolute inflammatory cell counts in peripheral blood (neutrophils, white blood cells, lymphocytes and monocytes) and ratios based on these cell counts (NLR, PLR and LMR) may play a key role in predicting the overall survival of patients with tumours, including colorectal cancer [19], head and neck cancer [20], non-small cell lung cancer [21]. Chen and his colleagues have shown that red cell distribution width (RDW) has predictive value for determining the survival of patients with oesophageal cancer [22]. Yusuke Ishibashi and his team believe that CAR is the most important predictor of OS in patients with oesophageal cancer [23]. A study by Hongdian Zhang and Xiaobin Shang et al. showed that systemic immune-inflammation index (SII) and prognostic nutritional index (PNI) are 
powerful indicators of invasive biology and poor prognosis in ESCC patients. The combination of SII and PNI can improve the accuracy of prognosis in patients with oesophageal cancer [24]. A retrospective analysis by Apostolos Gaitanidis and his colleagues showed that markers of the systemic inflammatory response are prognostic factors in patients with pancreatic neuroendocrine tumours [25]. Some previous studies have shown that inflammatory markers may affect the survival of cancer patients in many ways. Cancer-associated inflammation alters and polarizes the tumour microenvironment, and although it is not associated with tumour necrosis, it can increase the propensity for tumour recurrence and metastasis [26, 27]. Inflammatory cells can not only inhibit the proliferation and migration of tumour cells [28], but also eliminate residual tumour cells and micrometastases [29]. Lymphocytes play an important role in promoting anti-tumour immunity, and lymphopenia may impair the efficacy of the immune system. For example, if the level of effector $T$ cells is insufficient, cell-mediated cytotoxicity may be weakened, and the killing effect on tumour cells is also weakened [30].

In our study, it was demonstrated that LMR and NLR may be predictors of overall survival in patients with advanced oesophageal cancer after administration of chemoradiotherapy. In this study, we analysed the relationship between LMR and NLR and survival in patients with advanced oesophageal cancer undergoing chemoradiotherapy. First, we used a receiver operating characteristic curve (ROC) to analyse the optimal cutoff value for predicting the OS index, and the optimal cutoff value for LMR and NLR was 3.03 and 2.64, respectively, according to the ROC curve. Univariate and multivariate logistic regression analyses were then used to show that NLR (OR 2.233 (95\% CI 1.67-2.96), $p<0.05$ ) and LMR (OR 0.278 (95\% CI $0.205-0.376), \mathrm{p}<0.05)$ are closely related to the survival of patients with advanced oesophageal cancer receiving chemoradiotherapy. The analysis showed that the overall survival of patients with oesophageal cancer with an LMR less than 3.03 before treatment was significantly shorter than that of those with an LMR greater than 3.03. Moreover, the value of LMR before treatment was positively correlated with the survival of patients with oesophageal cancer. The value of NLR before treatment was negatively correlated with the survival of patients with oesophageal cancer, because the average survival time of patients with oesophageal cancer with an NLR greater than 2.64 and an NLR less than 2.64 was 10.3 months and 19.8 months, respectively. When we used the patient progression-free survival time as the secondary end point, we drew the same conclusion. At the same time, we performed a chi-square test analysis and found that LMR is related to clinical stage and treatment effect and that NLR is related to tumour location, $\mathrm{N}$ stage, clinical stage and treatment effect.

Although our data and results were accurately refined and calculated, this study has some limitations. First, only 204 patients were examined in this study, which may result in unstable results due to the small sample size. Second, TNM staging is only clinical staging, and although we used ultrasound endoscopy and contrastenhanced CT and PET/CT to determine staging, these methods may limit the ability to assess the prognosis of ESCC compared with pathological staging. Furthermore, our study only verifies the predictive value of LMR and NLR for patient survival, and we need to establish a systematic mathematical prediction model to serve the clinic. Finally, this was a single-centre and retrospective study; thus, all the included patients were from a single hospital, and the conclusions were not verified in other centres. Therefore, this study requires further prospective trials at multiple centres to confirm the reproducibility of the results in heterogeneous populations.

\section{Conclusion}

LMR and NLR are predictors of outcome for patients with advanced oesophageal cancer who receive concurrent chemoradiotherapy. The value of LMR and NLR can help clinicians predict the survival of patients and to select appropriate treatment schemes.

\section{Abbreviations \\ AUC: Area Under The Curve; Cl: Confidence Interval; CR: Complete Remission; CT: Computed Tomography; CTV: Clinical Target Volume; ESCC: Oesophageal Squamous Cell Carcinoma; GTV: Gross Target Volume; LMR: Lymphocyte-To- Monocyte Ratio; MRI: Magnetic Resonance Imaging; NLR: Neutrophil-To- Lymphocyte Ratio; ORR: Objective Response Rate; OS: Overall Survival; PET- CT: Positron Emission Tomography-Computed Tomography; PFS: Progression-Free Survival; PR: Partial Remission; PTV: Planning Target Volume; ROC: Receiver-Operating Characteristic}

\section{Acknowledgements \\ We appreciate the efforts of all members of our radiotherapy department and the support of all the patients' families.}

\section{Authors' contributions}

KLL was involved in data analysis, interpretation and manuscript writing. XFX, WHC, MS and $\mathrm{HZ}$ were involved in collection and assembly of data. CLZ was involved in conception and design. All authors read and approved the final manuscript.

\section{Funding}

Financial support was received from the Natural Science Foundation of Zhejiang Province, China (No. LY15H160063). The funds were used to pay for the follow-up work, the purchase of statistical software, the learning of some statistical methods, and the purchase and reading of some references.

\section{Availability of data and materials}

The dataset in this study is available by request from the corresponding author. For more information, please contact the corresponding author.

Ethics approval and consent to participate

All the procedures followed were in accordance with the ethical guidelines of the Helsinki Declaration. The study protocol was approved by the Medical 
Ethical Committee of the First Affiliated Hospital of Wenzhou Medical University. Because all the patients in this retrospective study had died, informed consent was obtained from their family members or their pretreatment authorized recipients

\section{Consent for publication}

Not applicable.

\section{Competing interests}

The authors declare that they have no competing interests.

\section{Author details}

'Wenzhou Medical University, Wenzhou 325000, People's Republic of China. ${ }^{2}$ Department of Radiotherapy and Chemotherapy, The First Affiliated Hospital of Wenzhou Medical University, WenZhou, China.

Received: 2 May 2019 Accepted: 13 September 2019

Published online: 26 October 2019

\section{References}

1. Siegel RL, Miller KD, Jemal A. Cancer statistics, 2015. CA Cancer J Clin. 2015; 65(1):5-29.

2. $\mathrm{M} \mathrm{A}$, et al. Global incidence of oesophageal cancer by histological subtype in 2012. Gut. 2015;64(3):381-7.

3. W C, et al. Cancer statistics in China, 2015. CA Cancer J Clin. 2016;66(2):115-32.

4. F K, S E, M N. Current trends in multimodality treatment of esophageal and gastroesophageal junction cancer - review article. Surg Oncol. 2017;26(3): 290-5.

5. $\quad \mathrm{V}, \mathrm{V}$., M. AC, and L. SH, Advances in radiotherapy Management of Esophageal Cancer. J Clin Med, 2016. 5(10): p. undefined.

6. Martini $\mathrm{S}$, et al. Volumetric modulated arc therapy (VMAT) in the treatment of esophageal cancer patients. Med Oncol. 2018;35(12):150.

7. Yang GQ, et al. Intensity-modulated radiotherapy at high-volume center improves survival in patients with esophageal adenocarcinoma receiving trimodality therapy. Dis Esophagus. 2019;32(8).

8. Nicolini $\mathrm{G}$, et al. Volumetric modulation arc radiotherapy with flattening filter-free beams compared with static gantry IMRT and 3D conformal radiotherapy for advanced esophageal cancer: a feasibility study. Int J Radiat Oncol Biol Phys. 2012;84(2):553-60.

9. Warren $\mathrm{S}$, et al. Potential of proton therapy to reduce acute hematologic toxicity in concurrent Chemoradiation therapy for esophageal Cancer. Int J Radiat Oncol Biol Phys. 2017;99(3):729-37.

10. Xi M, et al. Comparative outcomes after definitive Chemoradiotherapy using proton beam therapy versus intensity modulated radiation therapy for esophageal Cancer: a retrospective, single-institutional analysis. Int J Radiat Oncol Biol Phys. 2017:99(3):667-76.

11. Chen YK, et al. Plasma matrix metalloproteinase 1 improves the detection and survival prediction of esophageal squamous cell carcinoma. Sci Rep. 2016;6:30057.

12. Gusella M, et al. Genetic prediction of long-term survival after neoadjuvant chemoradiation in locally advanced esophageal cancer. Pharmacogenomics J. 2017:17(3):252-7.

13. Yuan $\mathrm{D}$, et al. The preoperative neutrophil-lymphocyte ratio predicts recurrence and survival among patients undergoing RO resections of adenocarcinomas of the esophagogastric junction. J Surg Oncol. 2014; 110(3):333-40

14. Hirahara $\mathrm{N}$, et al. A novel prognostic scoring system using inflammatory response biomarkers for esophageal squamous cell carcinoma. World J Surg. 2018;42(1):172-84.

15. Conway AM, et al. Significance of blood neutrophil-to-lymphocyte ratio for prognostic stratification of patients with gastroesophageal junction adenocarcinoma in the era of the 8th edition of the American joint committee on Cancer (AJCC8) staging. Med Oncol. 2017;34(6):116.

16. Toss MS, et al. The prognostic significance of lysosomal protective protein (cathepsin a) in breast ductal carcinoma in situ. Histopathology. 2019;74(7): 1025-35.

17. QG X, et al. A novel HBx genotype serves as a preoperative predictor and fails to activate the JAK1/STATs pathway in hepatocellular carcinoma. J Hepatol. 2019;70(5):904-17.

18. Mase $\mathrm{S}$, et al. ZNF671 DNA methylation as a molecular predictor for the early recurrence of serous ovarian cancer. Cancer Sci. 2019;110(3):1105-16.
19. Chan JC, et al. The lymphocyte-to-monocyte ratio is a superior predictor of overall survival in comparison to established biomarkers of Resectable colorectal Cancer. Ann Surg. 2017;265(3):539-46.

20. Charles KA, et al. Systemic inflammation is an independent predictive marker of clinical outcomes in mucosal squamous cell carcinoma of the head and neck in oropharyngeal and non-oropharyngeal patients. BMC Cancer. 2016;16:124.

21. Sekine $K$, et al. Change in the lymphocyte-to-monocyte ratio is an early surrogate marker of the efficacy of nivolumab monotherapy in advanced non-small-cell lung cancer. Lung Cancer. 2018;124:179-88.

22. Chen GP, et al. A nomogram to predict prognostic value of red cell distribution width in patients with esophageal Cancer. Mediat Inflamm. 2015:2015:854670.

23. Ishibashi $Y$, et al. Prognostic value of preoperative systemic Immunoinflammatory measures in patients with esophageal Cancer. Ann Surg Oncol. 2018;25(11):3288-99.

24. Zhang $\mathrm{H}$, et al. The predictive value of a preoperative systemic immuneinflammation index and prognostic nutritional index in patients with esophageal squamous cell carcinoma. J Cell Physiol. 2019;234(2):1794-802

25. Gaitanidis A, et al. Markers of systemic inflammatory response are prognostic factors in patients with pancreatic neuroendocrine tumors (PNETs): a prospective analysis. Ann Surg Oncol. 2018:25(1):122-30.

26. Chechlinska M, Kowalewska M, Nowak R. Systemic inflammation as a confounding factor in cancer biomarker discovery and validation. Nat Rev Cancer. 2010;10(1):2-3.

27. Brandau S, Dumitru CA, Lang S. Protumor and antitumor functions of neutrophil granulocytes. Semin Immunopathol. 2012;35(2):163-76.

28. Bastid J, et al. Lymphocyte-derived interleukin-17A adds another brick in the wall of inflammation-induced breast carcinogenesis. Oncoimmunology. 2014:3:e28273

29. Nazir T, et al. Lymphocytopenia; induced by vinorelbine, doxorubicin and cisplatin in human cancer patients. Breast Dis. 2015;35(1):1-4

30. Xiao WW, et al. A low lymphocyte-to-monocyte ratio predicts unfavorable prognosis in pathological T3N0 rectal Cancer patients following Total Mesorectal excision. J Cancer. 2015;6(7):616-22.

\section{Publisher's Note}

Springer Nature remains neutral with regard to jurisdictional claims in published maps and institutional affiliations.
Ready to submit your research? Choose BMC and benefit from:

- fast, convenient online submission

- thorough peer review by experienced researchers in your field

- rapid publication on acceptance

- support for research data, including large and complex data types

- gold Open Access which fosters wider collaboration and increased citations

- maximum visibility for your research: over $100 \mathrm{M}$ website views per year

At $\mathrm{BMC}$, research is always in progress.

Learn more biomedcentral.com/submissions 